Musées, Patrimoine et Culture scientifiques et techniques

$176 \mid 2018$

mars-avril 2018

\title{
Développer le dialogue entre les différents acteurs du monde muséal
}

\section{Audrey Doyen et Cindy Lebat}

\section{OpenEdition \\ Journals}

Édition électronique

URL : http://journals.openedition.org/ocim/2058

DOI : 10.4000/ocim.2058

ISSN : 2108-646X

Éditeur

OCIM

Édition imprimée

Date de publication : 1 mars 2018

Pagination : $32-33$

ISSN : 0994-1908

Référence électronique

Audrey Doyen et Cindy Lebat, « Développer le dialogue entre les différents acteurs du monde muséal », La Lettre de I'OCIM [En ligne], 176 | 2018, mis en ligne le 01 mars 2019, consulté le 10 octobre 2020. URL : http://journals.openedition.org/ocim/2058 ; DOI : https://doi.org/10.4000/ocim.2058

Ce document a été généré automatiquement le 10 octobre 2020

Tous droits réservés 


\title{
Développer le dialogue entre les différents acteurs du monde muséal
}

\author{
Audrey Doyen et Cindy Lebat
}

\section{Comment est né ce projet?}

Le 26 septembre 2017 se tenait dans la salle du conseil de la Maison de la Recherche de l'université Paris 3-Sorbonne Nouvelle un débat sur les mutations professionnelles dans le secteur de la culture et plus particulièrement des musées spécialement en lien avec l'actualité politique, économique et sociale française. Réunissant des intervenants issus du monde académique (Fabien van Geert, maître de conférence en muséologie à l'université Paris 3-Sorbonne Nouvelle), du ministère de la Culture et de la Communication (Susana Muston, chargée de mission à la Direction générale des patrimoines) et des musées (Nicolas Liucci-Goutnikov, conservateur du musée national d'Art moderne), cette première réunion lançait un évènement régulier : les Rencontres muséo.

Depuis deux ans, un groupe de jeunes chercheurs de l'université Paris 3-Sorbonne Nouvelle se réunit de manière informelle pour partager actualités, bibliographies et questionnements autour des musées. Ces rencontres mensuelles intéressent bien vite un cercle plus large - d'abord cons-

titué de doctorants d'horizons divers, puis de chercheurs confirmés, et enfin de professionnels de musées. Sentant le besoin de se réunir entre professionnels de musées pour parler de thématiques parfois sensibles, comme les enjeux et la gestion du mécénat au sein des institutions en février 2016, nous avons décidé avec Lina Uzlyte (chargée de cours et doctorante à l'université Paris 3-Sorbonne Nouvelle) de professionnaliser et de structurer davantage ces rencontres.

Quels sont les objectifs de l'association Mêtis à l'origine du projet?

L'association Mêtis, née en septembre 2017, a pour ambition de créer et renforcer, au sein de l'univers des musées, le lien entre recherche et terrain, d'inscrire dans la durée un dialogue nécessaire entre disciplines académiques et corps de métier, d'initier et de promouvoir des espaces de partages entre différentes pratiques 
artistiques et culturelles. Au sein de l'association, nous revendiquons trois axes comme valeurs fortes :

- la centralité du contact humain et l'intérêt pour l'engagement sensible : au-delà des plateformes numériques ou des carnets d'adresses bien remplis, mais impersonnels, l'association valorise le contact humain, les rencontres de visu, afin de personnaliser les rapports professionnels et de tisser des liens ;

- un regard sur le monde culturel tel qu'il se développe et se vit aujourd'hui à l'heure actuelle : l'association, dans une visée fédératrice, veut donner de la visibilité aux larges réseaux d'acteurs impliqués dans les musées et à leurs professions. Du concepteur au visiteur en passant par le médiateur et le chercheur, Mêtis s'engage à poser un regard critique sur le monde culturel en devenir, en considérant avec lucidité ses enjeux politiques, économiques et sociaux ;

- une expertise scientifique rigoureuse, portée par les trois chercheuses fondatrices de Mêtis, avec toute l'exigence, la précision et l'excellence que celle-ci implique : discussions et actions commentées, bibliographies, ressources.Mêtis désire autant participer à développer la recherche qu'à la transmettre.

Comment se déroulent les Rencontres muséo ?

Pour l'organisation des Rencontres muséo, nous bénéficions du soutien du Labex ICCA (Industries culturelles et création artistique), de l'université Paris 3-Sorbonne Nouvelle et du laboratoire Cerlis (Centre de recherche sur les liens sociaux). Structurée en trois cycles thématiques, eux-mêmes divisés en trois séances, la première saison des Rencontres muséo s'étend jusqu'à juin 2018. Jusqu'ici, les thématiques abordées ont porté sur la fatigue muséale, de l'influence du lieu sur l'expérience de visite, de la non-présence du numérique dans certains musées, ou de la place du son dans les expositions ${ }^{1}$.

La dernière séance de chaque cycle est consacrée à une application pratique du sujet soulevé, sous la forme d'une visite-expérimentation dans un musée partenaire ; la visite du premier cycle, sur le thème de l'expérience de visite, s'est déroulée au musée du quai Branly-Jacques Chirac (voir l'encadré ci-contre).

\section{La visite-expérimentation au musée du quai Branly-Jacques Chirac}

Le premier cycle des Rencontres Muséo avait pour objectif de questionner "l'expérience du visiteur", à travers la conjugaison des discours, réflexions et exemples présentés et discutés avec les participants lors de deux tables rondes. Organisée le 19 décembre 2017, la visite-expérimentation, marquant la fin de ce cycle, a été conçue comme un moyen de poursuivre de façon concrète et appliquée ces questionnements. Elle a amené une vingtaine de participants, à travers des expériences sensorielles et des méthodes originales de recueil de données, à interroger leur rapport à l'espace du musée, mais aussi aux œuvres et à leur message.

Cette visite a présenté un fort intérêt tant au niveau méthodologique que pour les pistes de réflexion qui en ont émergé, faisant notamment état d'une pluralité des registres de l'expérience. Les expériences des participants - fondées sur l'écoute de soi, la cartographie des émotions et le dessin - leur ont permis de prendre conscience et d'exprimer différents registres d'appréhension d'une exposition : 
cognitif, sensoriel, émotionnel. Il ressort en outre une prédominance globale du registre cognitif dans l'expérience de visite, qui a tendance à "écraser" les autres modalités expérientielles possibles, c'est-à-dire les formes de rencontre avec le musée. Cette visite a constitué une forme novatrice de recherche-action, alliant expérimentation, engagement de soi et retour réflexif sur ses propres pratiques.

\section{Que retenir de ces initiatives à mi-parcours du programme de cette saison?}

La participation importante aux rencontres témoigne d'un réel besoin d'un espace de discussion ouvert à tous les acteurs du monde muséal pour leur permettre de s'exprimer sur l'actualité et les possibilités d'évolution et de changements du domaine. En outre, les rencontres régulières, organisées de façon à laisser le plus possible de place à l'informel, au partage et à la discussion, ont permis à un réseau de se structurer encore plus fortement.

Aussi, le concept de visite-expérimentation et l'approche pratique de la recherche ont trouvé un public favorable et attentif, comme le montre le retour d'expérience de la visite organisée au musée du quai Branly-Jacques Chirac. Il témoigne de l'intérêt à trouver des applications concrètes à la recherche, à prendre du recul sur les concepts et développements théoriques pour s'engager dans des applications pratiques de nos réflexions.

II s'agit aussi de diffuser le plus largement possible les contenus des Rencontres?

En effet, l'association Mêtis s'applique à diffuser et transmettre des contenus des Rencontres, voire plus largement des recherches associées. Cette initiative participe d'une volonté plus large de diffuser les recherches académiques en cours. Observant un fort désir des acteurs du monde muséal de se tenir informés, mais conscientes de l'opacité parfois rédhibitoire de l'organisation de l'information et des structures académiques, nous avons développé quelques outils de diffusion des Rencontres.

Ainsi, chaque conférencier est interrogé pendant deux minutes sur une question un peu provocante, l'interview étant ensuite accessible sur la chaine Youtube des Rencontres; des comptes rendus ou extraits choisis de chaque rencontre sont diffusés sur les réseaux sociaux et le site internet de l'association; le site internet regroupe les principaux fruits de chaque recherche et les présentations ou ressources des conférenciers sont mises à la disposition des membres adhérents de l'association. En même temps, ces derniers participent au développement de ces ressources par leur soutien annuel.

Quelles sont les perspectives de développement de cet outil?

BIBLIOGRAPHIE

Fortes de ce bilan, nous sommes déterminées à développer Mêtis et les Rencontres Muséo. La plusvalue importante des visites-expérimentation sera mise en exergue la saison prochaine, puisque le concept sera développé sous forme de journées entières, permettant davantage d'échanges et 
rencontres. De façon à garder un rendez-vous ponctuel permettant aux personnes de se rencontrer et d'échanger sur leurs projets ou pratiques en cours, les Rencontres muséo continueront à se tenir tous les mois de manière informelle, et mettront en exergue un projet ou une actualité de l'un des membres.

Souhaitant répondre au besoin d'un pôle de ressources pour les professionnels du musée, à l'image de l'Ocim, Mêtis s'appliquera à diffuser et à transmettre les informations relatives à chaque rencontre et à produire un contenu de qualité, basé sur les recherches en cours, pour que tous les acteurs du monde muséal puissent être à la pointe de l'actualité des recherches.

Contact : www.metis.lab.com

\section{NOTES}

1. Pour consulter le programme et les ressources mises en ligne voir www.metis.lab.com

\section{RÉSUMÉS}

Fondatrices de l'association Mêtis à l'origine des Rencontres Muséo, Audrey Doyen et Cindy Lebat expliquent comment elles ont créé un espace de discussion pour permettre aux professionnels des musées d'échanger et de s'informer sur l'actualité et l'évolution des pratiques.

\section{INDEX}

Mots-clés : musées, espace d'échange, pratiques professionnelles

\section{AUTEURS}

\section{AUDREY DOYEN}

ethnologue et muséologue, doctorantes de l'université Paris 3-Sorbonne Nouvelle

\section{CINDY LEBAT}

spécialiste des enjeux et de l'appréhension des publics des musées en situation de handicap, doctorantes de l'université Paris 3-Sorbonne Nouvelle 